\title{
Aplicação de um Programa de Cessação do Tabagismo com Rodoviários Urbanos
}

Smoking Cessation Program Held In An Urban Bus Company

Aplicación De Un Programa De Cese Del Tabaquismo Con Empleados Del Transporte Urbano

Fernanda Machado

Lopes, Ana Carolina Peuker \& Lisiane Bizarro Rio Grande do Sul

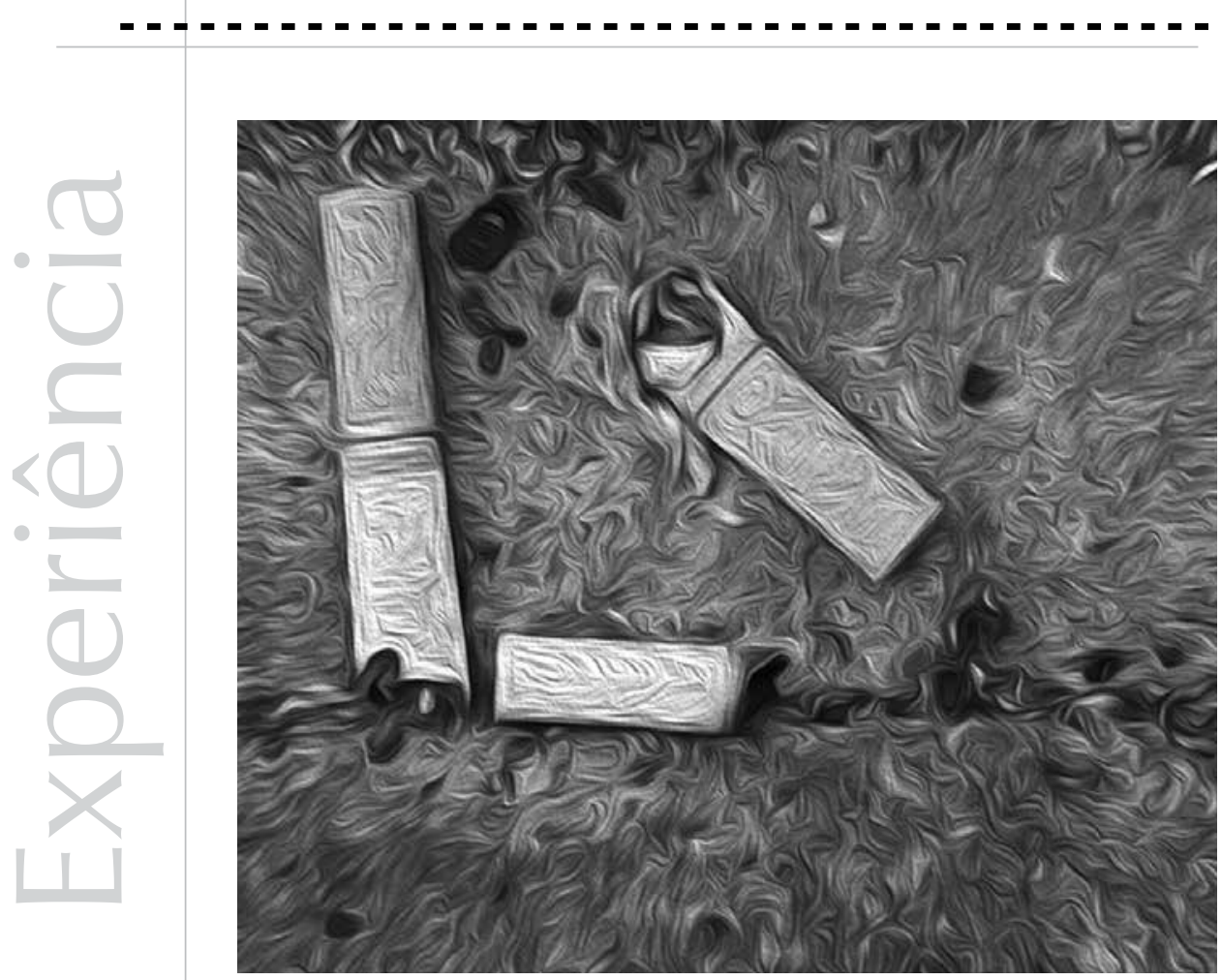


Resumo: O presente relato tem como objetivo descrever a aplicação de um programa de cessação do tabagismo realizado em uma empresa de transporte coletivo urbano que implantou a política do ambiente livre do fumo. Pretende-se contribuir apresentando um modelo de intervenção de abordagem cognitivocomportamental possível de ser implantado no contexto organizacional. Método: o programa foi realizado em três etapas: levantamento no número de fumantes ( $n=90 ; 13 \%$ do universo total de funcionários), avaliação do nível de dependência de nicotina ( $n=30 ; 33 \%$ dos fumantes) e realização dos grupos de cessação do tabagismo, dos quais participaram 16 fumantes (18\%). Os grupos aconteceram em quatro encontros semanais, com duração de duas horas cada, sendo que, após trinta dias, foi realizado um encontro de manutenção. Resultados: o programa demonstrou boa eficácia, pois, dos 16 fumantes que participaram, 7 atingiram a abstinência e 9 reduziram o consumo. Conclusão: os psicólogos podem ser agentes comprometidos em manter um ambiente livre de fumo e prevenir os efeitos do tabagismo nas organizações utilizando intervenções cognitivo-comportamentais como a descrita.

Palavras-chave: Tabagismo. Psicologia organizacional. Terapia cognitivo-comportamental. Saúde mental.

Abstract: The aim of this report is to describe a smoking cessation program held in an urban bus company which implemented a smoke-free environment policy. The aim of the program was to offer cognitivebehavioral counseling to workers who wished to quit smoking, facilitating the transition to a smoke-free environment and reducing healthcare costs associated with smoking for both the employee and the employer. Method: the program was conducted in three stages: assessing the number of smokers ( $n=90 ; 13 \%$ of total employees), assessing their level of nicotine dependence ( $n=30 ; 33 \%$ of smokers) and offering smoking cessation assistance consisting of education and counseling in groups $(n=16 ; 13 \%$ smokers agreed to participate). There were four weekly meetings in the company, lasting two hours each, and a fifth meeting thirty days later as a follow-up. Results: the program was efficient, as 7 quitted smoking and 9 reduced their consumption. Conclusion: psychologists can be comitted to maintain a smoke-free environment and the effect of smoking in organizations using planned cognitive-behavioral interventions similar to the one described in this report.

Keywords: Smoking. Organizational psychology. Cognitive-behavioral therapy. Mental health.

Resumen: El presente relato tiene como objetivo describir la aplicación de un programa de cese del tabaquismo realizado en una empresa de transporte colectivo urbano que implantó la política del ambiente libre del humo. Se desea contribuir presentando un modelo de intervención de abordaje cognoscitivo comportamental posible de ser implantado en el contexto organizacional. Método: el programa fue realizado en tres etapas: cómputo del número de fumadores ( $n=90 ; 13 \%$ del universo total de empleados), evaluación del nivel de dependencia de nicotina $(n=30 ; 33 \%$ de los fumadores) y realización de los grupos de cese del tabaquismo, de los cuales participaron 16 fumadores (18\%). Los grupos ocurrieron en cuatro encuentros semanales, con duración de dos horas cada uno, siendo que, después de treinta días, fue realizado un encuentro de mantenimiento. Resultados: el programa demostró una buena eficacia, pues, de los 16 fumadores que participaron, 7 alcanzaron la abstinencia y 9 redujeron el consumo. Conclusión: los psicólogos pueden ser agentes comprometidos en mantener un ambiente libre de humo y prevenir los efectos del tabaquismo en las organizaciones utilizando intervenciones cognoscitivo comportamentales como la descripta.

Palabras clave: Tabaquismo. Psicología organizacional. Terapia cognitiva-conductista. Salud mental.

Existem razões específicas que tornam o ambiente de trabalho um espaço propício para intervenções direcionadas à promoção da saúde, entre elas, o amplo espectro de alcance das intervenções, visto a grande e estável população exposta a ela, a maior chance de realizar um recrutamento adequado e a possibilidade de fortalecer o compromisso com a mudança de comportamento através da rede de apoio social estabelecida com colegas de trabalho (Task Force on Community Preventive
Services - TFCPS, 2010). Diversos estudos têm sido realizados no sentido de estimar perdas na produtividade e nos custos financeiros para os empregadores causados pelo tabagismo entre trabalhadores. Os prejuízos decorrentes do aumento do absenteísmo, da redução na produtividade e do tempo improdutivo no trabalho (causado pelas pausas para fumar) revelam significativas diferenças entre os funcionários fumantes e os não fumantes (Brunn, Stave, Downs, Alvir, \& Dirani, 2006; Tsai, Wen, Hu, Cheng, \& Huang, 2005). 
O uso do tabaco possui alta incidência entre trabalhadores e está associado a inúmeros riscos à saúde, como câncer, enfisema pulmonar, infarto do miocárdio, bronquite crônica, disfunção erétil, hipertensão arterial $e$ convulsão

(Figlie, Bordin, \& Laranjeira, 2004).
De acordo com estatísticas do Instituto Nacional do Seguro Social (INSS), os transtornos mentais, incluindo o tabagismo, ocupam a 3a posição entre as causas de concessão de benefício previdenciário, como auxílio-doença, afastamento do trabalho e aposentadorias por invalidez (Ministério da Saúde do Brasil, 2001). Nos últimos anos, percebe-se um crescente interesse por questões relacionadas à saúde/doença mental do trabalhador, pois comportamentos de risco, como, por exemplo, o tabagismo, representam um alto custo social e econômico, à medida que elevam a busca pelos serviços de saúde (Glina, Rocha, Batista, \& Mendonça, 2001). O número de organizações que oferecem programas de promoção de saúde para seus colaboradores cresceu consideravelmente nos Estados Unidos nas últimas duas décadas. Em 1991, esse percentual aproximava-se de $81 \%$; já em 2000, subiu para 90\% (TFCPS, 2010).

Iniciativas que busquem estimular a cessação do tabagismo constituem a regra de ouro para reduzir os custos relacionados à saúde, uma vez que a doença é considerada a principal causa de morte evitável em todo o mundo. $\mathrm{O}$ tabaco, especialmente quando usado como cigarro, é uma das drogas mais poderosas em termos de capacidade de gerar dependência; apesar disso, é uma substância psicoativa lícita, consumida por cerca de um terço da população brasileira (Carlini, Galduróz, Silva, Noto, \& Fonseca, 2006). O uso do tabaco possui alta incidência entre trabalhadores e está associado a inúmeros riscos à saúde, como câncer, enfisema pulmonar, infarto do miocárdio, bronquite crônica, disfunção erétil, hipertensão arterial e convulsão (Figlie, Bordin, \& Laranjeira, 2004).

O controle do tabagismo no ambiente de trabalho visa a reduzir as doenças e as mortes relacionadas ao uso do tabaco, além dos demais problemas que concorram para a má qualidade de vida do trabalhador e a diminuição da produtividade na empresa. Políticas de restrição de consumo em ambientes de trabalho impactam em taxas de cessação de fumar na ordem de 10 a $20 \%$ em um ano, sendo que $95 \%$ dos ex-fumantes abandonaram o hábito após auxílio de campanhas educativas e/ou aconselhamentos e intervenções motivacionais por profissionais da área da saúde (Instituto Nacional do Câncer - INCA, 1996). Nesse contexto, um número crescente de instituições tem implantado programas de promoção da saúde, como, por exemplo, as políticas de ambiente livre do fumo. A saúde do trabalhador está vinculada à maior produtividade e a menores custos; por esse motivo, um programa de controle e combate ao tabagismo beneficia tanto o empregador, que lucra ao ter um funcionário saudável, mais produtivo e menos dispendioso, quanto o empregado, que se beneficia em ter maior atenção à sua saúde e sua força de trabalho maximizada. Em consequência, a empresa é favorecida ao ter um ambiente eficiente e agradável.

Contudo, o tabagismo é uma doença, e o fumante necessita de apoio, aconselhamento, acompanhamento e tratamento médicosocial. Uma imposição coercitiva por parte de quem implanta a política de ambiente livre do tabaco não é indicada, pois os trabalhadores necessitam de orientações e de encorajamento para vencer o hábito de fumar por decisão própria. Assim, o psicólogo que intervém com o sofrimento dos trabalhadores deve considerar a subjetividade do indivíduo, seus valores, crenças e os significados que atribui a determinadas situações em um contexto social a fim de promover mudanças nas condições e nos processos de trabalho, com enfoque no âmbito coletivo (Santos, Goulart Júnior, Canêo, Lunardelli, \& Carvalho, 2010).

A abordagem cognitivo-comportamental é o tratamento não farmacológico para o tabagismo de maior eficácia, e tem sido 
oferecido pelo Ministério da Saúde à população em geral. O Instituto Nacional de Câncer (INCA) desenvolveu o Programa de Controle do Tabagismo e outros Fatores de Risco de Câncer, e, em parceira com as Secretarias Municipais de Saúde e Educação, coordenam o programa nas unidades de saúde e capacitam profissionais em ambientes de trabalho e escolas a desenvolvê-lo (Ministério da Saúde, 2001). A abordagem cognitivo-comportamental objetiva favorecer mudanças no estilo de vida do paciente bem como modificações de crenças e de comportamentos relacionados ao fumar. Esse tipo de abordagem é breve e focal, e assume como premissa que o comportamento de uso de drogas é aprendido, desencadeado e mantido por emoções e situações específicas, o que requer a modificação das crenças disfuncionais, a resolução de problemas e o reajuste social para a modificação do comportamento desadaptativo (Presman, Carneiro, \& Gigliotti, 2005).

O presente relato tem como objetivo descrever a implantação de um programa de cessação do tabagismo (PCT) para rodoviários (motoristas e cobradores) fumantes, funcionários de uma empresa de transporte urbano de Porto Alegre, RS, que adotou um ambiente livre do fumo. A modalidade de grupo favorece o relacionamento entre os participantes, a troca de experiências em relação ao histórico do tabagismo e a interpretação da realidade vivenciada. Além disso, visa a conduzi-los a tomar a decisão de parar de fumar a partir de informações sobre a doença com orientação, aconselhamento e acompanhamento de um profissional da saúde.

\section{Método}

\section{Participantes}

Através de um levantamento inicial realizado pela empresa, foi detectada uma prevalência de 90 funcionários (13\%) fumantes, todos do sexo masculino. Dentre eles, 30 (33\% dos fumantes) se inscreveram e responderam ao questionário de avaliação do nível de dependência de nicotina (FTND), mas nem todos compareceram aos encontros do PCT. Participaram efetivamente do programa 16 funcionários (18\% dos fumantes), que foram divididos em dois grupos de oito participantes, considerando o turno inverso das suas atividades laborais (ver fluxograma na Figura 1). A Tabela 1 apresenta a descrição da amostra em relação a idade, número de cigarros fumados por dia, tempo de uso do cigarro e nível de dependência de nicotina. Em relação ao nível de escolaridade, $69 \%$ dos participantes possuíam ensino médio completo e $31 \%$ ensino fundamental completo.

Tabela 1. Amostra em relação a idade, número de cigarros fumados por dia, tempo de uso do cigarro e nível de dependência de nicotina

\begin{tabular}{lcc}
\hline & Média & $\mathrm{dp}$ \\
\hline $\begin{array}{l}\text { Idade (anos) } \\
\text { Nível de dependência }\end{array}$ & 38 & 9,6 \\
$\begin{array}{l}\text { de nicotina } \\
\text { Quantidade de }\end{array}$ & 4,5 & 1,5 \\
$\begin{array}{l}\text { cigarros/dia } \\
\text { Tempo de uso de cigarro }\end{array}$ & 21 & 2,6 \\
& 19 & 8,8 \\
\hline
\end{tabular}

\section{Instrumentos}

Para avaliar o nível de monóxido de carbono (CO) dos fumantes, foi utilizado o monoxímetro (Smokerlyser), aparelho portátil que mede o no ar expelido através de expiração bucal posterior a uma apneia de 15 segundos. Os níveis de $\mathrm{CO}$ são medidos em partículas por milhão (ppm), e, em geral, alcançam em torno de $4 p p m$ no ar ambiente. Valores de 0 a $10 \mathrm{ppm}$ podem ser obtidos em pessoas não fumantes, valores entre $11 \mathrm{e}$ 20 ppm, em fumantes moderados, e valores 
entre 21 e 100 ppm, em fumantes graves (Bedfont, 1993). Esse aparelho foi usado somente para medir a abstinência após o programa, pois, durante o PCT, ele não estava disponível para uso das psicólogas por problemas técnicos.

Para avaliar a severidade da dependência da nicotina, foi utilizado o Fagerström Test for Nicotine Dependence (FTND) (Heatherton, Kozlowski, Frecker, \& Fagerström, 1991). Nesse estudo, escores até quatro foram considerados baixa dependência, e escores maiores ou iguais a cinco, dependência alta. A versão utilizada é amplamente aplicada no Brasil, e foi avaliada como confiável na avaliação da dependência de tabaco em diferentes contextos e populações (MenesesGaya, Zuardi, Loureiro, \& Crippa, 2009).

\section{Procedimentos}

Etapa 1: Levantamento do número de fumantes da empresa

Participaram 642 (94\%) dos 680 funcionários ativos da empresa. O levantamento foi feito através de um formulário no qual constavam as perguntas: Você é fumante? Você gostaria de ajuda para parar de fumar? Esse formulário foi aplicado pessoalmente pela psicóloga da empresa, que fazia as perguntas oralmente e as registrava por escrito, no dia da folha de pagamento. Declaram-se fumantes 90 funcionários $(13 \%)$, dos quais $70(10 \%)$ afirmaram desejar ajuda para parar de fumar, e que receberam um convite personalizado para avaliarem seu nível de dependência de nicotina no setor de Recursos Humanos e se inscreverem no Programa de Cessação do Tabagismo.

Etapa 2: Avaliação do nível de dependência dos fumantes
A avaliação foi realizada através do monoxímetro e do Fagerström Test for Nicotine Dependence (FTND). Após a avaliação, os 30 funcionários se inscreveram no Programa de Cessação do Tabagismo, conforme mostra a Figura 1.

Etapa 3: Realização dos grupos

O objetivo dos grupos era oferecer psicoeducação e treino de estratégias cognitivas e comportamentais para o manejo dos sintomas de abstinência e da fissura.

Etapa 4: Encontro de manutenção

O encontro de manutenção aconteceu 30 dias após o último encontro do grupo, e teve como objetivo reforçar as estratégias cognitivas e comportamentais para o manejo dos sintomas de abstinência e da fissura.

Os grupos foram coordenados por duas psicólogas, uma vinculada à empresa e outra externa, e os encontros foram realizados na sala de treinamentos da empresa, equipada com recurso de data show e cadeiras estofadas. Cada encontro teve duração total de $2 \mathrm{hs}$ e frequência semanal, totalizando quatro encontros, que foram realizados no turno inverso ao de trabalho. Assim, foram realizados dois grupos: um no turno da manhã e outro à tarde. Foi organizada uma apostila com resumos ilustrados dos tópicos principais abordados em cada encontro, que foi desenvolvida a partir do material disponibilizado pelo Ministério da Saúde e pelo Instituto Nacional do Câncer (Abordagem de Tratamento do Tabagismo na Rede SUS, 2004), além de explicações sobre as técnicas cognitivo-comportamentais ensinadas. Cada participante recebeu uma apostila com os assuntos discutidos no grupo. 
ETAPA 1- Levantamento dos Fumantes

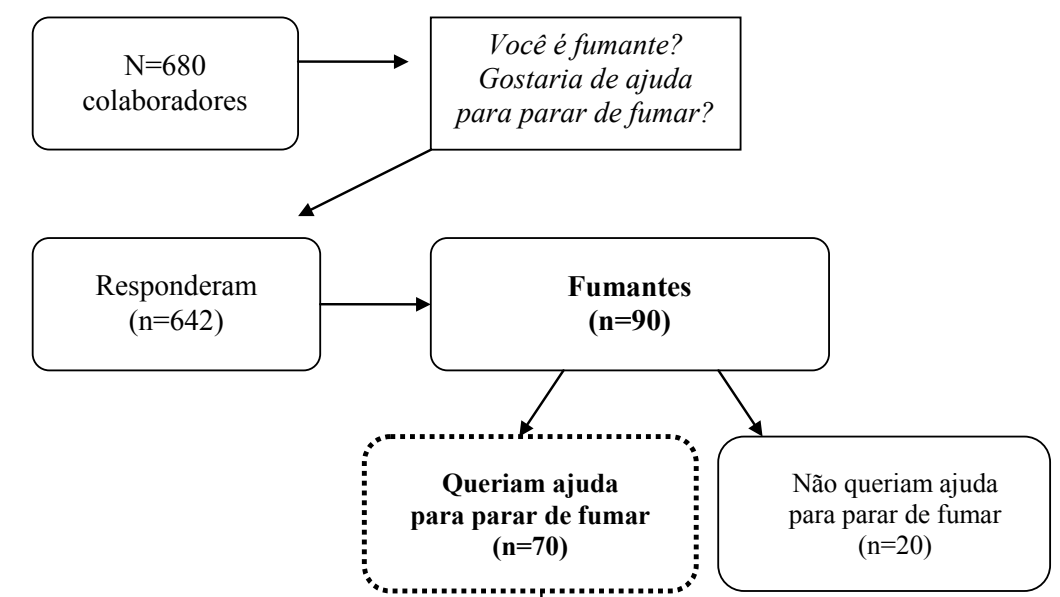

ETAPA 2- Avaliação do nível dependência nicotina e inscrição no programa

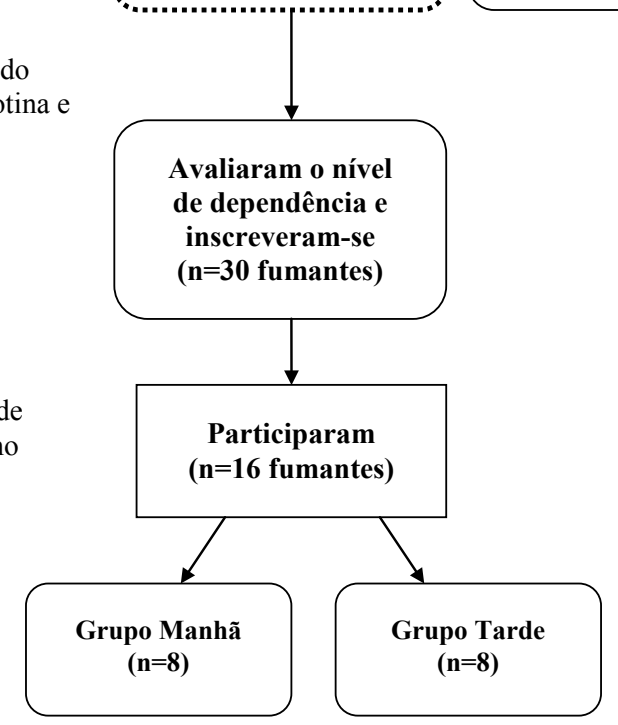
ETAPA 3- Programa de
Cessação do Tabagismo ETAPA 3- Programa de
Cessação do Tabagismo Avaliaram o nível
de dependência e
inscreveram-se
( $\mathrm{n}=\mathbf{3 0}$ fumantes)

Figura 1. Fluxograma das etapas do programa

Os tópicos discutidos em cada encontro foram:

10 encontro: padrão de consumo dos participantes, ambivalência (porque fumar e porque parar), prejuízos à saúde provocados pelo tabagismo e dependência química, psicológica e comportamental. 
$2^{\underline{0}}$ encontro: métodos para deixar de fumar (parada abrupta e gradual), síndrome de abstinência, exercícios de relaxamento e técnica de resolução de problemas. Essa técnica foi ensinada com o objetivo de instrumentalizar os fumantes a lidarem com os problemas de modo mais assertivo (identificar o problema, listar soluções possíveis, avaliar prós e contras das soluções, eleger e implementar a melhor opção), para ampliar seu repertório e evitar a recaída em momentos de estresse.

3o encontro: manejo da fissura, aumento de peso, exame de pensamentos e crenças sobre o tabaco e rede de apoio. O manejo da fissura incluiu a técnica da distração e diversas técnicas cognitivas (ex. substituir pensamentos positivos a respeito do cigarro, como "um cigarro agora me relaxaria", por negativos "um cigarro agora me deixaria fedorento e com dificuldade pra respirar") e comportamentais (fazer uma higiene bucal completa e demorada imediatamente após as refeições).

4 o encontro: prevenção de recaída, cartão de enfrentamento e estratégias de enfrentamento para situações de risco. O cartão de enfrentamento consiste em confeccionar um cartão, de tamanho adequado para poder carregar consigo, no qual constem as razões mais fortes para manter-se sem fumar, ou seja, deve ter frases ou imagens de forte impacto para impedi-lo de fumar. As estratégias de enfrentamento incluem o planejamento de o que fazer quando e onde estiver em uma situação em que possa fumar e local de risco. O fumante faz uma tabela descrevendo o que fará em cada situação que identifica como risco (ex.: "No aniversário do meu chefe, quando ele me convidar para sair pra fumar, direi que parei de fumar e irei ao banheiro lavar o rosto, ler meu cartão de enfrentamento e fazer um telefonema").

\section{Resultados}

Considerando que o número de inscritos no PCT foi de 30 fumantes e que 16 efetivamente participaram dos quatro encontros, o índice de retenção foi de $53 \%$. Apesar de a média do nível de dependência de nicotina ter sido moderada (FTND $=4,5$ pontos), 8 deles pontuaram nível de dependência elevada. Em relação ao índice de abstinência, dentre os 16 fumantes que participaram do programa, 7 (44\% dos participantes) atingiram a abstinência (comprovada por medição do monoxímetro, no qual apresentaram níveis de não fumantes), e 9 (56\% dos participantes) reduziram o consumo do tabaco. Porém, no acompanhamento de 30 dias após o término do programa, 4 dos 9 participantes que haviam reduzido o fumo (44\%) retornaram ao antigo padrão de consumo; portanto, os participantes que optaram pelo método da parada abrupta foram mais beneficiados pelo programa do que os que escolheram o método da parada gradual. Não houve correlações entre aqueles que ficaram abstinentes e as variáveis tempo como fumante, quantidade de cigarros fumados por dia, nível de dependência de nicotina e turno de trabalho.

No último encontro e no encontro de manutenção, os principais benefícios relatados pelos participantes que conseguiram atingir a abstinência foram uma sensação de controle, sensação de liberdade, elogios dos familiares, melhora nas relações sociais e fim do preconceito. Além desses, benefícios referentes à melhora da saúde, como aumento da capacidade pulmonar, melhora do paladar e do olfato foram citados tanto pelos participantes que atingiram a abstinência como pelos participantes que apenas tinham conseguido a redução. 


\section{Discussão/conclusões}

Pode-se considerar que o programa desenvolvido obteve êxito, uma vez que todas as etapas planejadas foram cumpridas e os objetivos iniciais propostos foram alcançados. A etapa do levantamento foi de fundamental importância tanto para que a empresa pudesse ter um panorama geral do número de fumantes como para sensibilizá-los a fazer uma tentativa de parar de fumar. Observou-se, de modo geral, que os participantes apresentavam um repertório comportamental empobrecido, com déficits importantes de recursos de enfrentamento (habilidades de coping) e de habilidades sociais. Foram discutidos tópicos relativos a essas questões, incluindo treino de resolução de problemas e de assertividade.

No que se refere à escolha do método de parada, abrupta ou gradual, os fumantes do presente relato que obtiveram maior sucesso na obtenção e na manutenção da abstinência após 30 dias foram os que escolheram o método da parada abrupta. Porém, um artigo que revisou os princípios e a efetividade das técnicas não farmacológicas para o tratamento do tabagismo (Presman et al., 2005) verificou que a maioria dos estudos sobre os métodos de parada não mostra diferença significativa de eficácia. Contrariando um pouco a recomendação da maioria dos profissionais de saúde de preferir a parada abrupta em vez da gradual, as autoras enfatizaram no artigo de revisão que o fundamental é que a escolha do paciente seja respeitada. Contudo, os participantes do presente relato que optaram pela parada abrupta obtiveram maior êxito na abstinência em médio prazo (após 30 dias) do que aqueles que optaram pela parada gradual.

Pesquisas sobre a eficácia de intervenções que ajudam pessoas a parar de fumar revelam que qualquer técnica é melhor do que nenhuma, seja aconselhamento médico, terapia comportamental, material de autoajuda, modalidade individual ou grupal; são cautelosos, porém, em recomendar uma em relação à outra, pois poucos estudos comparam a eficácia entre abordagens (Lancaster, Stead, Silagy, \& Sowden, 2000; Presman et al., 2005). O estudo de revisão das técnicas psicossociais utilizadas como tratamentos não farmacológicos para o tabagismo revelou que tanto aconselhamento médico como aconselhamento telefônico e terapia comportamental se mostraram eficazes; contudo, destacaram que, na maioria das revisões sistemáticas e metanálises que estudam terapias comportamentais, a taxa de abstinência após seis meses varia de 15\% a 25\% (Presman et al., 2005).

A eficácia do tratamento comportamental pode ser atribuída, em parte, à utilização de diversas técnicas no tratamento do tabagismo, como o treino de habilidades e de resolução de problemas, relaxamento, assertividade e prevenção de recaída. Além disso, o tratamento em grupo pode proporcionar algumas vantagens específicas, como maior suporte social e maior diversidade na discussão sobre fatores de risco de recaída, estratégias para enfrentar situações difíceis e manejo de fissura (Presman et al., 2005). No grupo aqui relatado, as coordenadoras estimularam os participantes a criar uma rede de apoio entre eles (ex: trocas de experiências, criação de lista de contato telefônico, etc.) com a qual pudessem obter apoio não só durante mas também após o término do tratamento.

Uma sugestão importante para futuros programas de cessação do tabagismo é que se faça um acompanhamento individual concomitante à realização do grupo. O tratamento individual adapta as técnicas às características específicas e às particularidades dos hábitos de cada fumante (Presman et al., 2005). Programas de cessação do 
tabagismo administrados em múltiplos formatos (individual e grupal) aumentam as taxas de abstinência, e, portanto, devem ser encorajados (Fiore, 2000).

No presente estudo, entre o momento da inscrição e do primeiro encontro, o índice de adesão foi de 53\%. Funcionários que se inscreveram e não compareceram aos encontros confessaram que desistiram de participar porque pensaram que a abordagem seria similar à do aconselhamento médico, com foco principal nos prejuízos à saúde. Contudo, sabe-se que a ambivalência é um dos aspectos presentes no comportamento aditivo, e um fator fundamental para o êxito do tratamento é a motivação para a mudança (Melo, Oliveira, \& Ferreira, 2006). Além disso, o fato de o tratamento ter sido oferecido pela própria empresa pode também ter despertado crenças ambivalentes, como "meu emprego está em risco se eu não parar de fumar" versus "a empresa está preocupada com minha saúde", o que pode ser considerado uma limitação deste estudo.

Uma revisão da literatura sobre estudos que avaliaram programas de promoção da saúde no ambiente de trabalho revelou que a simples mensuração de índices de saúde, mesmo quando acompanhada de feedback, não constitui a melhor abordagem para promover a mudança de comportamento e a redução dos riscos de saúde entre os funcionários de uma empresa (Lancaster et al., 2000). A intervenção deve abranger avaliações ao longo do tempo (follow-up) que ampliem a percepção do empregado acerca dos riscos de saúde aos quais está exposto e sessões que desenvolvam habilidades necessárias à mudança de comportamento e potencializem a motivação para adotar um estilo de vida mais saudável. Ademais, as oportunidades no contexto organizacional para a prática de hábitos saudáveis devem ser ampliadas.
Contudo, a implantação de um programa bem sucedido não pode prescindir de uma adequada avaliação inicial, pois assim pode-se evitar o dispêndio de recursos e o aumento dos gastos com cuidados de saúde. Entre as melhores práticas, destaca-se o desenvolvimento de programas comprometidos com a organização, que forneçam incentivos para os colaboradores participarem, que estabeleçam triagens e screenings de necessidades adequados, que baseiem suas intervenções em evidências, que tenham um processo efetivo de implantação e que ainda contem com um programa contínuo de avaliação (TFCPS, 2010). Assim, uma limitação do programa aqui relatado foi o tempo de acompanhamento após o término do PCT. Realizou-se um seguimento breve de 30 dias, mas sabe-se que o ideal é que se realizem estudos com delineamento longitudinal que obtenham resultados de seguimento mais longos (ex. após seis meses e um ano) (TFCPS, 2010). Por último, sugere-se ainda que outras medidas (por exemplo, motivação para a mudança, comorbidades psiquiátricas) sejam utilizadas antes, durante e após a intervenção. Tais medidas poderão contribuir para o ajuste do PCT às necessidades dos participantes, resultando em maior adesão e remissão do tabagismo e, em consequência, na redução dos custos de saúde, tanto para o empregado quanto para o empregador. 


\section{Fernanda Machado Lopes}

Doutora em Psicologia pela Universidade Federal do Rio Grande do Sul e Professora Substituta da

Universidade Federal do Rio Grande do Sul, Porto Alegre - RS - Brasil.

E-mail: femlopes23@gmail.com

\section{Ana Carolina Peuker}

Pós-doutora em Psicologia pela Universidade Federal do Rio Grande do Sul, Porto Alegre - RS - Brasil.

E-mail: acpeuker@hotmail.com

\section{Lisiane Bizarro}

Pós-doutora em Psicologia pelo Institute Of Psychiatry King's College London e Professora Adjunta da Universidade Federal do Rio Grande do Sul, Porto Alegre - RS - Brasil.

E-mail: lisiane.bizarro@gmail.com

Endereço para envio de correspondência:

Rua Ramiro Barcellos 2600. CEP: 90035-003. Porto Alegre, RS

Recebido: 11/12/2011, 1a Reformulação: 14/12/2012, Aprovado: 22/01/2013

\section{Referências}

Bedfont, S. (1993). Operator's manual for mini and micro smokerlyzers. Upchurch: Bedfont Scientific.

Brunn, W. B., Stave, G. M., Downs, K. E., Alvir, J. M., \& Dirani, R. (2006). Effect of smoking status on productivity loss. Journal of Occupational and Environmental Medicine, 48(10), 1099-1108.

Carlini, E. A., Galduróz, J., Silva, A., Noto, A., \& Fonseca, A. (2006). II levantamento domiciliar sobre o uso de drogas psicotrópicas no Brasil: estudo envolvendo as 108 maiores cidades do país. São Paulo: CEBRID - Centro Brasileiro de Informações sobre Drogas Psicotrópicas /UNIFESP Universidade Federal de São Paulo.

Figlie, N. B., Bordini, S., \& Laranjeira, R. (2004). Aconselhamento em dependência química. São Paulo: Roca.

Fiore, M. C. (2000). US public health service clinical practice guideline: Treating tobacco use and dependence. Respiratory Care, 45(10), 1200-1262.

Glina, D. M. R., Rocha, L. E., Batista, M. L., \& Mendonça, M. G. V. (2001). Saúde mental e trabalho: uma reflexão sobre o nexo com o trabalho e o diagnóstico, com base na prática. Cadernos de Saúde Pública, 17(3), 607-616.

Heatherton, T. F., Kozlowski, L. T., Frecker, R. C., \& Fagerström, K. O. (1991). The Fagerström test for nicotine dependence: A revision of the Fagerström Tolerance Questionnaire. British Journal of Addiction, 86, 1119-1127.

Instituto Nacional de Câncer - INCA (1996). Programa Prevenção Sempre: módulo Tabagismo.

(Manual da Comissão Executiva para implantação do Programa em Ambientes de Trabalho). Recuperado em 10 jul. 2009 de www.se.gov.br/.../anexo_16_tabagismo_manual_comisso_ executiva_programa_pr.doc.

Instituto Nacional de Câncer. Coordenação de Prevenção e
Vigilância - Conprev. (2004). Deixando de fumar sem mistérios. Rio de Janeiro: MS/INCA.

Lancaster, T., Stead, L., Silagy, C., \& Sowden, A. (2000). Effectiveness of interventions to help people stop smoking: Findings from the Cochrane Library. British Medical Journal, 321, 355-358.

Melo, W., Oliveira, M., \& Ferreira, E. (2006). Estágios motivacionais, sintomas de ansiedade e de depressão no tratamento do tabagismo. Interação em Psicologia, 10, 91-99.

Meneses-Gaya, I. C., Zuardi, A. W., Loureiro, S. R., \& Crippa, J. A. (2009). As propriedades psicométricas do Teste de Fagerström para Dependência de Nicotina. Jornal Brasileiro de Pneumologia, 35, 73-82.

Ministério da Saúde. (2001). Doenças relacionadas ao trabalho: Manual de Procedimentos para os Serviços de Saúde. Brasília, DF: Autor.

Presman, S., Carneiro, E., \& Gigliotti, A. (2005). Tratamentos não-farmacológicos para o tabagismo. Revista de Psiquiatria Clínica, 32(5), 267-275.

Santos, L. C., Goulart Júnior, E., Canêo, L. C., Lunardelli, M. C. F., \& Carvalho, P. L. T. (2010). Psicologia e profissão: neurose profissional e a atuação do psicólogo organizacional frente à questão. Psicologia: Ciência e Profissão, 30(2), 248-261.

Task Force on Community Preventive Services. (2010). Recommendations for worksite-based interventions to improve worker's health. American Journal of Preventive Medicine, $38,232-236$.

Tsai, S. P., Wen, C. P., Hu, S. C., Cheng, T. Y., \& Huang, S. J. (2005). Workplace smoking related absenteeism and productivity costs in Taiwan. Tobacco Control, 14(1), 33-37. 